$3-25-2020$

\title{
A New Liu Type of Estimator for the Restricted SUR Estimator
}

Kristofer Månsson

Jönköping University, kristofer.mansson@ju.se

B. M. Golam Kibria

Florida International University, kibriag@fiu.edu

Ghazi Shukur

Linnæus University, ghazi.shukur@Inu.se

Follow this and additional works at: https://digitalcommons.wayne.edu/jmasm

Part of the Applied Statistics Commons, Social and Behavioral Sciences Commons, and the Statistical Theory Commons

\section{Recommended Citation}

Månsson, K., Kibria, B. M. G., \& Shukur, G. (2019). A new Liu type of estimator for the restricted SUR estimator. Journal of Modern Applied Statistical Methods, 18(1), eP2758. doi: 10.22237/jmasm/ 1556669340

This Regular Article is brought to you for free and open access by the Open Access Journals at DigitalCommons@WayneState. It has been accepted for inclusion in Journal of Modern Applied Statistical Methods by an authorized editor of DigitalCommons@WayneState. 


\section{A New Liu Type of Estimator for the Restricted SUR Estimator}

\author{
Kristofer Månsson \\ Jönköping University \\ Jönköping, Sweden
}

\author{
B. M. Golam Kibria \\ Florida International University \\ Miami, FL
}

\author{
Ghazi Shukur \\ Linnæus University \\ Växjö, Sweden
}

A new Liu type of estimator for the seemingly unrelated regression (SUR) models is proposed that may be used when estimating the parameters vector in the presence of multicollinearity if the it is suspected to belong to a linear subspace. The dispersion matrices and the mean squared error (MSE) are derived. The new estimator may have a lower MSE than the traditional estimators. It was shown using simulation techniques the new shrinkage estimator outperforms the commonly used estimators in the presence of multicollinearity.

Keywords: $\quad$ Estimation, MSE, multicollinearity, SUR, Liu, simulation

\section{Introduction}

In estimating demand and production functions in economics or patient data over time, and similar applications, consider the system of equations to be estimated:

$$
\mathbf{Y}_{i}=\mathbf{X}_{i} \boldsymbol{\beta}_{i}+\boldsymbol{\varepsilon}_{i}, \quad i=1, \ldots, M
$$

where $\mathbf{Y}_{i}$ is a $T \times 1$ vector of observations on the dependent variable, $\mathbf{X}_{i}$ is a $T \times(p+1)$ design matrix including the independent variables and the intercept (i.e. a vector off ones) and $\boldsymbol{\varepsilon}_{i}$ is a $T \times 1$ vector that includes the error term which is assumed to be normally distributed. Furthermore, we have that $M$ is the number of equations in the system, $T$ the number of observations, and $p$ the number of explanatory variables. 1 If the error terms of the different equations are correlated then the most efficient way of estimating the above system of equations is seemingly unrelated regression (SUR) method suggested by Zellner (1962). Using this method, define $\mathbf{Y}=\left[\mathbf{Y}_{1}, \ldots, \mathbf{Y}_{M}\right]^{\prime}, \mathbf{X}=\left[\mathbf{X}_{1}, \ldots, \mathbf{X}_{M}\right]^{\prime}$, and $\boldsymbol{\varepsilon}=\left[\boldsymbol{\varepsilon}_{1}, \ldots, \boldsymbol{\varepsilon}_{M}\right]^{\prime}$. Define

doi: 10.22237/jmasm/1556669340 | Accepted: April 11, 2018; Published: March 25, 2020.

Correspondence: Kristofer Månsson, kristofer.mansson@ju.se 


\section{MÅNSSON ET AL}

$\mathbf{P}:=\left(\hat{\boldsymbol{\sigma}}^{-1 / 2} \otimes \mathbf{I}\right)$, where $\hat{\boldsymbol{\sigma}}:=\sum_{t=1}^{T} \hat{\boldsymbol{\varepsilon}}_{t} \hat{\boldsymbol{\varepsilon}}_{t} / T$ and is estimated by applying the standard ordinary least squares (OLS) estimator on each of the $i^{\text {th }}$ equations in (1) and then calculate the residual series. Then, estimate

$$
\mathbf{P Y}=\mathbf{P X \beta}+\mathbf{P \varepsilon}
$$

using the standard OLS equation

$$
\hat{\boldsymbol{\beta}}_{\mathrm{SUR}}=\left(\mathbf{X}^{*} \cdot \mathbf{X}^{*}\right)^{-1} \mathbf{X}^{* \prime} \mathbf{Y},
$$

where $\mathbf{X}^{*}=\mathbf{P X}$ and $\mathbf{Y}^{*}=\mathbf{P Y}$. However, often when estimating system of equations we have that $\boldsymbol{\beta}$ may belong to a linear subspace defined by $\mathbf{R} \boldsymbol{\beta}=\mathbf{r}$, where $\mathbf{R}$ is a $q \times p$ matrix of full rank with $q<p$ and $\mathbf{r}$ is a $q \times 1$ vector. A restricted estimator could be used instead. This may occur, for example in an applied demand system, where it is one assumed there is no change in consumer demand if all prices change by the same proportion. The restricted SUR estimator discussed in Srivastava and Giles (1987) could be used:

$$
\hat{\boldsymbol{\beta}}_{\mathrm{SUR}}^{\mathbf{R}}=\hat{\boldsymbol{\beta}}_{\mathrm{SUR}}+\left(\mathbf{X}^{*} \mathbf{X}^{*}\right)^{-1} \mathbf{R}^{\prime}\left(\mathbf{R} \mathbf{X}^{*} \mathbf{X}^{*} \mathbf{R}^{\prime}\right)^{-1}\left(\mathbf{r}-\mathbf{R} \hat{\boldsymbol{\beta}}_{\mathrm{SUR}}\right)
$$

A problem when estimating the parameters of this type of model is multicollinearity. This may for example occur due to the fact that prices of different commodities are usually highly correlated with each other. Both $\hat{\boldsymbol{\beta}}_{\text {SUR }}$ and $\hat{\boldsymbol{\beta}}_{\text {SUR }}^{\mathbf{R}}$ are instable and they have a large variance. A potential remedy is to apply some shrinkage estimator and an attractive method is the Liu type of estimator proposed by Liu (1993). The Liu type of estimator combines then the advantage of the Stein estimator (the smoothing is a linear function of the smoothing parameter), and it has the advantage of the ridge regression (Hoerl \& Kennard, 1970a, b) estimator (i.e. that the smoothing of each element in $\hat{\boldsymbol{\beta}}_{\text {SUR }}$ is not identical). Therefore, it has become more popular and studied in many papers such as Akdeniz and Kaçiranlar (1995, 2001), Kaçiranlar et al. (1999), Kaçiranlar (2003), and Alheety and Kibria (2009), among others. The Liu type of estimator for SUR models is a generalization of the Liu type estimator (Liu, 1993) and may be defined as follows:

$$
\hat{\boldsymbol{\beta}}_{\mathrm{SUR}}^{\mathrm{Lu}}=\mathbf{F}_{d} \hat{\boldsymbol{\beta}}_{\mathrm{SUR}},
$$


where $\mathbf{F}_{d}=\left(\mathbf{X}^{* \prime} \mathbf{X}^{*}+\mathbf{I}\right)^{-1}\left(\mathbf{X}^{* \prime} \mathbf{X}^{*}+d \mathbf{I}\right)$ and $\mathbf{I}$ is the $M p \times M p$ identity matrix. In this paper we also suggest the following new restricted Liu type of estimator for SUR models in order to overcome the multicollinearity problem:

$$
\hat{\boldsymbol{\beta}}_{\mathrm{SUR}}^{\mathbf{R}, \mathrm{Liu}}=\mathbf{F}_{d} \hat{\boldsymbol{\beta}}_{\mathrm{SUR}}^{\mathbf{R}}
$$

Therefore, the purpose of this study is to suggest a new restricted Liu type of estimator for SUR models.

\section{The MSE Properties of Different Estimators}

The MSE of any estimator $\hat{\boldsymbol{\beta}}$ for estimating $\boldsymbol{\beta}$ is defined as

$$
\operatorname{MSE}(\hat{\boldsymbol{\beta}})=\operatorname{E}(\hat{\boldsymbol{\beta}}-\boldsymbol{\beta})(\hat{\boldsymbol{\beta}}-\boldsymbol{\beta})^{\prime}=\mathrm{D}(\hat{\boldsymbol{\beta}})+(\operatorname{bias}(\hat{\boldsymbol{\beta}}))(\operatorname{bias}(\hat{\boldsymbol{\beta}}))^{\prime}
$$

where $\mathrm{D}(\hat{\boldsymbol{\beta}})$ is the dispersion matrix. We have the following dispersion matrices for SUR, Liu type estimator, restricted SUR and Liu type restricted SUR models, respectively:

$$
\begin{gathered}
\mathrm{D}\left(\hat{\boldsymbol{\beta}}_{\mathrm{SUR}}\right)=\mathbf{S}^{-1}, \\
\mathrm{D}\left(\hat{\boldsymbol{\beta}}_{\mathrm{SUR}}^{\mathrm{Liu}}\right)=\mathbf{F}_{d} \mathbf{S}^{-1} \mathbf{F}_{d}^{\prime}, \\
\mathrm{D}\left(\hat{\boldsymbol{\beta}}_{\mathrm{SUR}}^{\mathrm{R}}\right)=\mathbf{A}, \\
\mathrm{D}\left(\hat{\boldsymbol{\beta}}_{\mathrm{SUR}}^{\mathrm{R}, \mathrm{Liu}}\right)=\mathbf{F}_{d} \mathbf{A} \mathbf{F}_{d}^{\prime},
\end{gathered}
$$

where $\mathbf{S}=\mathbf{X}^{* \prime} \mathbf{X}^{*}, \quad \mathbf{A}=\mathbf{S}^{-1}-\mathbf{S}^{-1} \mathbf{R}^{\prime}\left(\mathbf{R S}^{-1} \mathbf{R}^{\prime}\right)^{-1} \mathbf{R} \mathbf{S}^{-1}$, and $\hat{\boldsymbol{\beta}}_{\mathrm{SUR}}$ and $\hat{\boldsymbol{\beta}}_{\mathrm{SUR}}^{\mathbf{R}}$ are unbiased estimators of $\boldsymbol{\beta}$. The MSE of the different estimators assuming the restriction actually holds are 


\section{MÅNSSON ET AL}

$$
\begin{gathered}
\operatorname{MSE}\left(\hat{\boldsymbol{\beta}}_{\mathrm{SUR}}\right)=\operatorname{tr}\left(\mathrm{D}\left(\hat{\boldsymbol{\beta}}_{\mathrm{SUR}}\right)\right)=\operatorname{tr}\left(\mathbf{S}^{-1}\right)=\sum_{j=1}^{J} \frac{1}{\lambda_{j}}, \\
\operatorname{MSE}\left(\hat{\boldsymbol{\beta}}_{\mathrm{SUR}}^{\mathrm{Liu}}\right)=\operatorname{tr}\left(\mathrm{D}\left(\hat{\boldsymbol{\beta}}_{\mathrm{SUR}}^{\mathrm{Liu}}\right)\right)+\operatorname{tr}\left(\left(\mathbf{F}_{d} \hat{\boldsymbol{\beta}}_{\mathrm{SUR}}-\boldsymbol{\beta}^{*}\right)\left(\mathbf{F}_{d} \hat{\boldsymbol{\beta}}_{\mathrm{SUR}}-\boldsymbol{\beta}^{*}\right)^{\prime}\right) \\
=\sum_{j=1}^{J} \frac{\left(\lambda_{j}+d\right)^{2}}{\lambda_{j}\left(\lambda_{j}+1\right)^{2}}+(d-1)^{2} \sum_{j=1}^{J} \frac{\alpha_{j}^{2}}{\left(\lambda_{j}+1\right)^{2}} \\
\operatorname{MSE}\left(\hat{\boldsymbol{\beta}}_{\mathrm{SUR}}^{\mathbf{R}}\right)=\operatorname{tr}\left(\mathrm{D}\left(\hat{\boldsymbol{\beta}}_{\mathrm{SUR}}^{\mathbf{R}}\right)\right)=\operatorname{tr}(\mathbf{A})=\sum_{j=1}^{J} b_{j j} \\
\operatorname{MSE}\left(\hat{\boldsymbol{\beta}}_{\mathrm{SUR}}^{\mathrm{R}, \mathrm{Liu}}\right)=\operatorname{tr}\left(\mathrm{D}\left(\hat{\boldsymbol{\beta}}_{\mathrm{SUR}}^{\mathrm{R}}\right)\right)+\operatorname{tr}\left(\left(\mathbf{F}_{d} \hat{\boldsymbol{\beta}}_{\mathrm{SUR}}^{\mathrm{R}}-\boldsymbol{\beta}^{*}\right)\left(\mathbf{F}_{d} \hat{\boldsymbol{\beta}}_{\mathrm{SUR}}^{\mathrm{R}}-\beta\right)^{\prime}\right) \\
=\sum_{j=0}^{J} \frac{\left(\lambda_{j}+d\right)^{2}}{\left(\lambda_{j}+1\right)^{2}} b_{j j}+(d-1)^{2} \sum_{j=0}^{J} \frac{\alpha_{j}^{2}}{\left(\lambda_{j}+1\right)^{2}}
\end{gathered}
$$

where $b_{j j}(j=1,2, \ldots, J)$ are the diagonal elements of $\mathbf{B}$ which may be defined as $\mathbf{B}=\mathbf{Q}^{\prime} \mathbf{A} \mathbf{Q}, \mathbf{Q}$ is defined as an orthogonal matrix such that $\mathbf{Q}^{\prime} \mathbf{S} \mathbf{Q}=\mathbf{\Lambda}$ with $\boldsymbol{\Lambda}=\operatorname{diag}\left(\lambda_{1}, \ldots, \lambda_{J}\right)$ and $\lambda_{1}, \ldots, \lambda_{J}$ the eigenvalues of the matrix $\mathbf{S}$, and $\alpha_{j}$ is the $j^{\text {th }}$ element of the vector $\boldsymbol{\alpha}=\mathbf{Q}^{\prime} \boldsymbol{\beta}^{*}$. By comparing the standard Liu estimator in equation (13) and the SUR estimator in equation (12), it is clear that it is possible to find a value of $d$ so that the decease of the variance is larger than the increase of due to the bias (as discussed in Liu, 1993). The completely new type of estimator in equation (15) also outperform the traditional restricted estimator in equation (14) using the same type of argument (i.e. the decrease of the variance exceeds the increase of the MSE due to the bias). Hence, there may be a substantial improvement using the new estimator, especially in the presence of multicollinearity. The estimation of $d$ is discussed in Liu (1993) where the optimal value is derived. The same type of estimator may be used here. 


\section{The Monte Carlo Simulation}

\section{The Design of the Experiment}

Consider the MSE of the different types of estimators in different empirically relevant situations to investigate the finite sample performance, even though asymptotically the Liu type of estimators will outperform the traditional methods. The core factor varied in the design of the experiment is the degree of correlation $\left(\rho^{2}\right)$ between the regressors. The strength of the correlation may be varied, and to generate the explanatory variables (for example, see Kibria, 2003; Kibria \& Banik, 2016):

$$
x_{t j i}=\left(1-\rho^{2}\right)^{1 / 2} z_{t j i}+\rho z_{t p i}, \quad t=1,2, \ldots, T ; j=1,2, \ldots, p ; i=1,2, \ldots, M,
$$

where $z_{t j i}$ are pseudo-random numbers from the standard normal distribution. Consider three different values of $\rho^{2}$ corresponding to $0.75,0.90$ and 0.99 . Use three equations in each system and we choose to generate models with two explanatory variables. The $n$ observations for the dependent variable are simulated using equation (1), where the error terms are generated using the equation $\boldsymbol{\varepsilon}_{t}=\mathbf{L} \boldsymbol{\eta}_{t}$, where $\boldsymbol{\eta}_{t} \sim$ iid $\mathrm{N}(0, \mathbf{I}), \mathbf{L L}^{\prime}=\boldsymbol{\Sigma}$, and $\boldsymbol{\Sigma}$ is a circulant* matrix (more precisely a Toeplitz matrix). The following parameter settings generate positive definite covariance matrixes:

$$
\Sigma_{3 \times 3}=\operatorname{circ}(0.5,0.5,0.2), \quad \Sigma_{3 \times 3}=\operatorname{circ}(1,0.5,0.2), \quad \Sigma_{3 \times 3}=\operatorname{circ}(3,0.5,0.2),
$$

where the variance of the error term is changed. The restrictions used are the following: The restrictions are set to the following for one restriction:

$$
\mathbf{R}=\left[\begin{array}{llllll}
1 & 0 & 1 & 0 & 1 & 0
\end{array}\right] \text { and } \mathbf{r}=[1]
$$

and the following for two restrictions:

$$
\mathbf{R}=\left[\begin{array}{cccccc}
1 & 0 & 1 & 0 & 1 & 0 \\
1 & -1 & 0 & 0 & 0 & 0
\end{array}\right] \text { and } \mathbf{r}=\left[\begin{array}{l}
1 \\
0
\end{array}\right]
$$

The experiment is replicated 10000 times by generating new pseudo-random numbers and then the MSE is estimated using the following formula: 


\section{MÅNSSON ET AL}

$$
\operatorname{MSE}=\frac{\sum_{r=1}^{10000}(\hat{\boldsymbol{\beta}}-\boldsymbol{\beta})^{\prime}(\hat{\boldsymbol{\beta}}-\boldsymbol{\beta})}{10000} .
$$

The simulated MSEs for different values of $d$ and $\rho$, for variances $0.5,1$, and 3 and for one restriction are presented in Tables $1-3$, respectively and for variances 0.5 , 1 , and 3 and for two restrictions in Tables 4-6, respectively.

Table 1. Results when residual variance is 0.5 and with 1 restriction

\begin{tabular}{|c|c|c|c|c|c|c|c|c|c|c|c|}
\hline \multirow[b]{2}{*}{$\rho$} & \multirow[b]{2}{*}{$n$} & \multicolumn{2}{|c|}{$d=1.00$} & \multicolumn{2}{|c|}{$d=0.75$} & \multicolumn{2}{|c|}{$d=0.50$} & \multicolumn{2}{|c|}{$d=0.25$} & \multicolumn{2}{|c|}{$d=0.00$} \\
\hline & & SUR & RSUR & Liu & RLiu & Liu & RLiu & Liu & RLiu & Liu & RLiu \\
\hline \multirow[t]{4}{*}{0.75} & 10 & 0.491 & 0.161 & 0.401 & 0.227 & 0.334 & 0.172 & 0.289 & 0.139 & 0.267 & 0.129 \\
\hline & 25 & 0.206 & 0.043 & 0.176 & 0.096 & 0.154 & 0.077 & 0.140 & 0.065 & 0.134 & 0.021 \\
\hline & 50 & 0.074 & 0.071 & 0.065 & 0.035 & 0.058 & 0.028 & 0.054 & 0.025 & 0.053 & 0.020 \\
\hline & 100 & 0.036 & 0.012 & 0.032 & 0.017 & 0.029 & 0.014 & 0.027 & 0.012 & 0.026 & 0.012 \\
\hline \multirow[t]{4}{*}{0.90} & 10 & 1.023 & 0.239 & 0.780 & 0.406 & 0.594 & 0.277 & 0.465 & 0.199 & 0.393 & 0.170 \\
\hline & 25 & 0.423 & 0.265 & 0.344 & 0.175 & 0.284 & 0.129 & 0.243 & 0.102 & 0.222 & 0.092 \\
\hline & 50 & 0.150 & 0.233 & 0.128 & 0.065 & 0.111 & 0.051 & 0.100 & 0.043 & 0.096 & 0.040 \\
\hline & 100 & 0.073 & 0.070 & 0.063 & 0.032 & 0.056 & 0.026 & 0.052 & 0.022 & 0.050 & 0.020 \\
\hline \multirow[t]{4}{*}{0.95} & 10 & 1.967 & 1.007 & 1.397 & 0.694 & 0.960 & 0.424 & 0.654 & 0.256 & 0.481 & 0.191 \\
\hline & 25 & 0.798 & 0.479 & 0.613 & 0.296 & 0.470 & 0.199 & 0.371 & 0.140 & 0.314 & 0.117 \\
\hline & 50 & 0.175 & 0.030 & 0.144 & 0.069 & 0.120 & 0.051 & 0.104 & 0.040 & 0.096 & 0.036 \\
\hline & 100 & 0.138 & 0.013 & 0.117 & 0.056 & 0.102 & 0.043 & 0.092 & 0.035 & 0.087 & 0.002 \\
\hline
\end{tabular}

Table 2. Results when residual variance is 1 and with 1 restriction

\begin{tabular}{|c|c|c|c|c|c|c|c|c|c|c|c|}
\hline \multirow[b]{2}{*}{$\rho$} & \multirow[b]{2}{*}{$n$} & \multicolumn{2}{|c|}{$d=1.00$} & \multicolumn{2}{|c|}{$d=0.75$} & \multicolumn{2}{|c|}{$d=0.50$} & \multicolumn{2}{|c|}{$d=0.25$} & \multicolumn{2}{|c|}{$d=0.00$} \\
\hline & & SUR & RSUR & Liu & RLiu & Liu & $\overline{\text { RLiu }}$ & Liu & RLiu & Liu & RLiu \\
\hline \multirow{4}{*}{0.75} & 10 & 1.179 & 0.406 & 0.934 & 0.598 & 0.746 & 0.436 & 0.615 & 0.331 & 0.541 & 0.281 \\
\hline & 25 & 0.502 & 0.135 & 0.417 & 0.265 & 0.353 & 0.206 & 0.310 & 0.168 & 0.288 & 0.152 \\
\hline & 50 & 0.185 & 0.176 & 0.157 & 0.098 & 0.137 & 0.079 & 0.125 & 0.067 & 0.119 & 0.063 \\
\hline & 100 & 0.091 & 0.017 & 0.078 & 0.049 & 0.069 & 0.040 & 0.063 & 0.034 & 0.061 & 0.032 \\
\hline \multirow[t]{4}{*}{0.90} & 10 & 2.537 & 3.650 & 1.869 & 1.094 & 1.352 & 0.707 & 0.986 & 0.453 & 0.770 & 0.332 \\
\hline & 25 & 1.084 & 0.299 & 0.858 & 0.486 & 0.683 & 0.344 & 0.560 & 0.250 & 0.489 & 0.205 \\
\hline & 50 & 0.397 & 0.072 & 0.330 & 0.184 & 0.280 & 0.140 & 0.247 & 0.112 & 0.230 & 0.100 \\
\hline & 100 & 0.190 & 0.011 & 0.161 & 0.090 & 0.139 & 0.071 & 0.125 & 0.058 & 0.119 & 0.053 \\
\hline \multirow[t]{4}{*}{0.95} & 10 & 4.899 & 2.586 & 3.394 & 1.877 & 2.233 & 1.092 & 1.415 & 0.582 & 0.942 & 0.346 \\
\hline & 25 & 2.080 & 1.722 & 1.560 & 0.832 & 1.154 & 0.534 & 0.862 & 0.337 & 0.685 & 0.242 \\
\hline & 50 & 0.764 & 0.323 & 0.615 & 0.324 & 0.501 & 0.231 & 0.422 & 0.170 & 0.376 & 0.140 \\
\hline & 100 & 0.365 & 0.062 & 0.304 & 0.159 & 0.257 & 0.119 & 0.226 & 0.093 & 0.210 & 0.052 \\
\hline
\end{tabular}




\section{LIU TYPE OF ESTIMATORS FOR THE RESTRICTED SUR ESTIMATOR}

Table 3. Results when residual variance is 3 and with 1 restriction

\begin{tabular}{|c|c|c|c|c|c|c|c|c|c|c|c|}
\hline \multirow[b]{2}{*}{$\rho$} & \multirow[b]{2}{*}{$n$} & \multicolumn{2}{|c|}{$d=1.00$} & \multicolumn{2}{|c|}{$d=0.75$} & \multicolumn{2}{|c|}{$d=0.50$} & \multicolumn{2}{|c|}{$d=0.25$} & \multicolumn{2}{|c|}{$d=0.00$} \\
\hline & & SUR & RSUR & Liu & RLiu & Liu & RLiu & Liu & RLiu & Liu & RLiu \\
\hline \multirow[t]{4}{*}{0.75} & 10 & 3.766 & 1.254 & 2.911 & 2.010 & 2.250 & 1.424 & 1.782 & 1.030 & 1.508 & 0.828 \\
\hline & 25 & 1.611 & 0.675 & 1.306 & 0.890 & 1.075 & 0.676 & 0.919 & 0.535 & 0.837 & 0.468 \\
\hline & 50 & 0.585 & 0.548 & 0.487 & 0.330 & 0.415 & 0.261 & 0.369 & 0.218 & 0.350 & 0.200 \\
\hline & 100 & 0.283 & 0.232 & 0.238 & 0.160 & 0.205 & 0.128 & 0.185 & 0.109 & 0.178 & 0.102 \\
\hline \multirow[t]{4}{*}{0.90} & 10 & 8.187 & 6.728 & 5.909 & 3.713 & 4.140 & 2.328 & 2.882 & 1.399 & 2.134 & 0.925 \\
\hline & 25 & 3.453 & 2.130 & 2.674 & 1.649 & 2.070 & 1.140 & 1.641 & 0.797 & 1.387 & 0.619 \\
\hline & 50 & 1.264 & 0.476 & 1.030 & 0.624 & 0.854 & 0.465 & 0.735 & 0.362 & 0.674 & 0.314 \\
\hline & 100 & 0.612 & 0.362 & 0.508 & 0.310 & 0.430 & 0.239 & 0.381 & 0.194 & 0.358 & 0.175 \\
\hline \multirow[t]{4}{*}{0.95} & 10 & 15.652 & 12.997 & 10.645 & 6.463 & 6.789 & 3.674 & 4.081 & 1.832 & 2.523 & 0.936 \\
\hline & 25 & 6.765 & 6.690 & 4.988 & 2.893 & 3.598 & 1.822 & 2.596 & 1.098 & 1.980 & 0.719 \\
\hline & 50 & 2.431 & 0.521 & 1.916 & 1.115 & 1.519 & 0.781 & 1.239 & 0.556 & 1.077 & 0.442 \\
\hline & 100 & 1.163 & 0.592 & 0.947 & 0.551 & 0.784 & 0.407 & 0.673 & 0.313 & 0.616 & 0.268 \\
\hline
\end{tabular}

Table 4. Results when residual variance is 0.5 and with 2 restrictions

\begin{tabular}{|c|c|c|c|c|c|c|c|c|c|c|c|}
\hline \multirow[b]{2}{*}{$\rho$} & \multirow[b]{2}{*}{$n$} & \multicolumn{2}{|c|}{$d=1.00$} & \multicolumn{2}{|c|}{$d=0.75$} & \multicolumn{2}{|c|}{$d=0.50$} & \multicolumn{2}{|c|}{$d=0.25$} & \multicolumn{2}{|c|}{$d=0.00$} \\
\hline & & SUR & RSUR & Liu & RLiu & Liu & RLiu & Liu & RLiu & Liu & RLiu \\
\hline \multirow[t]{4}{*}{0.75} & 10 & 0.400 & 0.183 & 0.333 & 0.137 & 0.289 & 0.110 & 0.267 & 0.103 & 0.267 & 0.129 \\
\hline & 25 & 0.174 & 0.078 & 0.152 & 0.061 & 0.139 & 0.051 & 0.132 & 0.049 & 0.134 & 0.061 \\
\hline & 50 & 0.065 & 0.029 & 0.058 & 0.023 & 0.054 & 0.020 & 0.053 & 0.019 & 0.053 & 0.023 \\
\hline & 100 & 0.031 & 0.014 & 0.028 & 0.011 & 0.026 & 0.010 & 0.026 & 0.009 & 0.026 & 0.012 \\
\hline \multirow[t]{4}{*}{0.90} & 10 & 0.774 & 0.354 & 0.587 & 0.234 & 0.458 & 0.162 & 0.387 & 0.138 & 0.393 & 0.170 \\
\hline & 25 & 0.341 & 0.152 & 0.281 & 0.109 & 0.240 & 0.084 & 0.219 & 0.076 & 0.222 & 0.092 \\
\hline & 50 & 0.126 & 0.055 & 0.110 & 0.042 & 0.100 & 0.035 & 0.095 & 0.032 & 0.096 & 0.040 \\
\hline & 100 & 0.064 & 0.028 & 0.056 & 0.022 & 0.052 & 0.018 & 0.050 & 0.017 & 0.050 & 0.020 \\
\hline \multirow[t]{4}{*}{0.95} & 10 & 1.349 & 0.612 & 0.925 & 0.366 & 0.630 & 0.214 & 0.464 & 0.157 & 0.481 & 0.191 \\
\hline & 25 & 0.621 & 0.268 & 0.477 & 0.176 & 0.376 & 0.120 & 0.318 & 0.099 & 0.314 & 0.117 \\
\hline & 50 & 0.237 & 0.105 & 0.199 & 0.076 & 0.172 & 0.058 & 0.159 & 0.051 & 0.096 & 0.036 \\
\hline & 100 & 0.117 & 0.052 & 0.101 & 0.039 & 0.091 & 0.031 & 0.086 & 0.029 & 0.087 & 0.032 \\
\hline
\end{tabular}

\section{Results}

From Tables 1 to 6, it is always the restricted Liu type estimator that outperforms the other estimators. In general, the values of $\rho$ increases, the MSEs also increase. It is always the traditional SUR estimator that has the highest MSE followed by the restricted SUR estimator. This pattern is especially clear for a high degree of multicollinearity. For larger sample sizes and for a low variation of the error term there is not much differences among estimators. However, they differ greatly for small sample sizes and large error variances. The restricted estimator and the Liu 


\section{MÅNSSON ET AL}

type estimator always have a lower estimated MSE than the traditional SUR method. Furthermore, the restricted Liu type estimator has a lower estimated MSE than the restricted estimator and the Liu type of estimator regardless of the situation evaluated. The Liu type estimators perform better for smaller shrinkage estimator $d$. The benefits are maximized for small sample sizes and when the variance of the error term is large. The new restricted Liu type of estimator is superior to the other type of estimators and may be recommended to use in empirical applications.

Table 5. Results when residual variance is 1 and with 2 restrictions

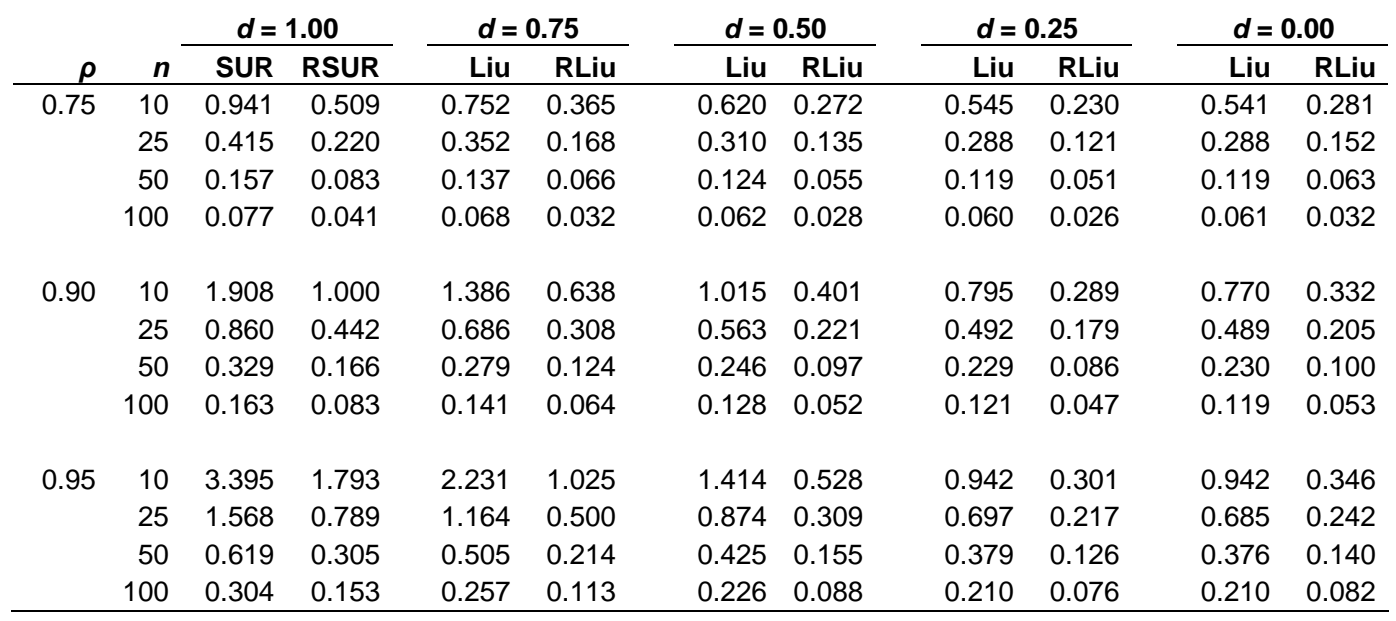

Table 6. Results when residual variance is 3 and with 2 restrictions

\begin{tabular}{|c|c|c|c|c|c|c|c|c|c|c|c|}
\hline \multirow[b]{2}{*}{$\rho$} & \multirow[b]{2}{*}{$n$} & \multicolumn{2}{|c|}{$d=1.00$} & \multicolumn{2}{|c|}{$d=0.75$} & \multicolumn{2}{|c|}{$d=0.50$} & \multicolumn{2}{|c|}{$d=0.25$} & \multicolumn{2}{|c|}{$d=0.00$} \\
\hline & & SUR & RSUR & Liu & RLiu & Liu & RLiu & Liu & RLiu & Liu & RLiu \\
\hline \multirow[t]{4}{*}{0.75} & 10 & 2.862 & 1.703 & 2.222 & 1.197 & 1.769 & 0.858 & 1.504 & 0.687 & 1.508 & 0.828 \\
\hline & 25 & 1.305 & 0.768 & 1.075 & 0.574 & 0.919 & 0.448 & 0.837 & 0.389 & 0.837 & 0.468 \\
\hline & 50 & 0.488 & 0.287 & 0.416 & 0.224 & 0.371 & 0.185 & 0.351 & 0.169 & 0.350 & 0.200 \\
\hline & 100 & 0.241 & 0.142 & 0.208 & 0.112 & 0.187 & 0.094 & 0.179 & 0.087 & 0.178 & 0.102 \\
\hline \multirow[t]{4}{*}{0.90} & 10 & 5.820 & 3.381 & 4.082 & 2.103 & 2.845 & 1.248 & 2.107 & 0.814 & 2.134 & 0.925 \\
\hline & 25 & 2.657 & 1.537 & 2.058 & 1.049 & 1.632 & 0.720 & 1.381 & 0.551 & 1.387 & 0.619 \\
\hline & 50 & 1.032 & 0.586 & 0.854 & 0.432 & 0.735 & 0.332 & 0.673 & 0.286 & 0.674 & 0.314 \\
\hline & 100 & 0.507 & 0.289 & 0.430 & 0.220 & 0.380 & 0.177 & 0.358 & 0.159 & 0.358 & 0.175 \\
\hline \multirow[t]{4}{*}{0.95} & 10 & 10.683 & 6.126 & 6.808 & 3.451 & 4.090 & 1.687 & 2.529 & 0.835 & 2.523 & 0.936 \\
\hline & 25 & 4.878 & 2.756 & 3.504 & 1.716 & 2.516 & 1.014 & 1.914 & 0.650 & 1.980 & 0.719 \\
\hline & 50 & 1.922 & 1.068 & 1.528 & 0.742 & 1.251 & 0.523 & 1.090 & 0.411 & 1.077 & 0.442 \\
\hline & 100 & 0.958 & 0.533 & 0.796 & 0.391 & 0.686 & 0.299 & 0.628 & 0.256 & 0.616 & 0.268 \\
\hline
\end{tabular}




\section{LIU TYPE OF ESTIMATORS FOR THE RESTRICTED SUR ESTIMATOR}

\section{Conclusion}

A new estimator is proposed for the SUR model when estimating the parameter vector and it is suspected that $\boldsymbol{\beta}$ may belong to a linear subspace defined by $\mathbf{R} \boldsymbol{\beta}=\mathbf{r}$. This is an empirically relevant situation and may be often in applied microeconomics when one wants to estimate demand functions such as the almost identical demand system (AIDS) or production functions for several countries or different industries such as the Cobb-Douglas equation. Other application could be to model the tourism demand or actuarial data. Hence, this new estimator has a wide variety of applications and may be used when the explanatory variables are highly inter-correlated. This new estimator has a lower MSE if there is such a value of the shrinkage parameter where the decrease of the variance is larger than the increase of the MSE. The new estimator outperforms the traditional estimators in finite samples, especially in small samples when the multicollinearity problem is severe.

\section{References}

Akdeniz, F., \& Kaçiranlar, S. (1995). On the almost unbiased generalized Liu estimator and unbiased estimation of the bias and MSE. Communications in Statistics - Theory and Methods, 24(7), 1789-1797. doi: 10.1080/03610929508831585

Akdeniz, F., \& Kaçıranlar, S. (2001). More on the new biased estimator in linear regression. Sânkhya: The Indian Journal of Statistics, Series B, 63(3), 321325.

Alheety, M. I., \& Kibria, B. M. G. (2009). On the Liu and almost unbiased Liu estimators in the presence of multicollinearity with heteroscedastic or correlated errors. Surveys in Mathematics and its Applications, 4, 155-167. Retrieved from http://www.utgjiu.ro/math/sma/v04/a13.html

Hoerl, A. E., \& Kennard, R. W. (1970a). Ridge regression: Application to nonorthogonal problems. Technometrics, 12(1), 69-82. doi:

10.1080/00401706.1970.10488635

Hoerl, A.E., \& Kennard, R.W. (1970b). Ridge regression: Biased estimation for nonorthogonal problems. Technometrics, 12(1), 55-67. doi:

10.1080/00401706.1970.10488634

Kaçiranlar, S. (2003). Liu estimator in the general linear regression model. Journal of Applied Statistical Science, 13, 229-234. 


\section{MÅNSSON ET AL}

Kaçiranlar, S., Sakallioğlu, S., Akdeniz, F., Styan, G. P. H., \& Werner, H. J. (1999). A new biased estimator in linear regression and a detailed analysis of the widely-analysed dataset on Portland cement. Sânkhya: The Indian Journal of Statistics, Series B, 61(3), 443-459.

Kibria, B.M.G. (2003). Performance of some new ridge regression estimators. Communications in Statistics - Theory and Methods 32(2), 419-435. doi: 10.1081/SAC-120017499

Kibria, B. M. G., \& Banik, S. (2016). Some ridge regression estimators and their performances. Journal of Modern Applied Statistical Methods. 15(1), 206238. doi: $10.22237 / \mathrm{jmasm} / 1462075860$

Liu, K. (1993). A new class of biased estimate in linear regression. Communications in Statistics - Theory and Methods, 22(2), 393-402. doi: 10.1080/03610929308831027

Zellner, A. (1962). An efficient method of estimating seemingly unrelated regression equations and tests for aggregation bias. Journal of the American Statistical Association, 57(298), 348-368. doi: 10.1080/01621459.1962.10480664 